\title{
CONTROLLABILITY OF SECOND ORDER SEMILINEAR ORDINARY DIFFERENTIAL SYSTEMS IN BANACH SPACES
}

\author{
K. BALACHANDRAN and S. MARSHAL ANTHONI \\ Bharathiar University \\ Department of Mathematics \\ Coimbatore 641046 , India
}

(Received January, 1998; Revised September, 1998)

\begin{abstract}
Sufficient conditions for controllability of semilinear second order ordinary differential systems in Banach spaces are established using the theory of strongly continuous cosine families. The results obtained are based on the Schaefer theorem.
\end{abstract}

Key words: Controllability, Semilinear Ordinary Differential System, The Schaefer Theorem.

AMS subject classifications: 93B05.

\section{Introduction}

Controllability of linear and nonlinear systems represented by ordinary differential equations in finite dimensional spaces has been extensively studied. Several authors extended the concept to infinite dimensional systems in Banach spaces with bounded operators. Chukwu and Lenhart [3] studied controllability of nonlinear systems in abstract spaces. Naito [7,8] studied controllability of semilinear systems and nonlinear Volterra integrodifferential systems. Quinn and Carmichael [11] showed that a controllability problem in Banach spaces can be converted into a fixed-point problem for a single-valued mapping. Balachandran et al. [1] established sufficient conditions for controllability of nonlinear integrodifferential systems in Banach spaces.

In many cases it is advantageous to treat second order abstract differential equations directly rather than convert them to first order systems. For example, Fitzgibbon [4] used the second order abstract differential equations for establishing the boundedness of solutions of an equation governing the transverse motion of an extensible beam. A useful tool for the study of abstract second order equations is the theory of strongly continuous cosine families. We will make use of some of the basic ideas from cosine family theory $[14,15]$. A motivation for second order systems can be found in [5, 6]. Recently, Park et al. [10] discussed controllability of second order nonlinear systems in Banach spaces with the help of the Schauder fixed point theorem. The purpose of this paper is to study controllability of semilinear second order differential systems in Banach spaces by using the Schaefer theorem. 


\section{Preliminaries}

We consider the semilinear second order control system

$$
\begin{gathered}
x^{\prime \prime}(t)=A x(t)+f\left(t, x(t), x^{\prime}(t)\right)+B u(t), \quad t \in J=[0, T] \\
x(0)=x_{0}, x^{\prime}(0)=y_{0}
\end{gathered}
$$

where the state $x(\cdot)$ takes values in a Banach space $X, x_{0}, y_{0} \in X, A$ is the infinitesimal generator of the strongly continuous cosine family $C(t)(t \in \mathbb{R})$, of bounded linear operators in $X, f$ is a nonlinear mapping from $J \times X \times X$ to $X, B$ is a bounded linear operator from $U$ to $X$ and the control function $u(\cdot)$ is given in $L^{2}(J, U)$, a Banach space of admissible control functions, with $U$ also being a Banach space.

Definition 1: [14] A one parameter family $C(t), t \in \mathbb{R}$, of bounded linear operators in the Banach space $X$ is called a strongly continuous cosine family iff

(i) $\quad C(s+t)+C(s-t)=2 C(s) C(t)$ for all $s, t \in \mathbb{R}$;

(ii) $\quad C(0)=I$;

(iii) $C(t) x$ is continuous in $t$ on $\mathbb{R}$ for each fixed $x \in X$.

Define the associated sine family $S(t), t \in \mathbb{R}$, by

$$
S(t) x=\int_{0}^{t} C(s) x d s, \quad x \in X, \quad t \in \mathbb{R} .
$$

Assume the following conditions on $A$.

$\left(H_{1}\right) \quad A$ is the infinitesimal generator of a strongly continuous cosine family $C(t), t \in \mathbb{R}$, of bounded linear operators from $X$ into itself and the adjoint operator $A^{*}$ is densely defined i.e., $\overline{D\left(A^{*}\right)}=X^{*}$ (see [2]).

The infinitesimal generator of a strongly continuous cosine family $C(t), t \in \mathbb{R}$, is the operator $A: X \rightarrow X$ defined by

where

$$
A x=\left.\frac{d^{2}}{d t^{2}} C(t) x\right|_{t=0}, x \in D(A)
$$

$$
D(A)=\{x \in X: C(t) x \text { is twice continuously differentiable in } t\}
$$

Define

$$
E=\{x \in X: C(t) x \text { is once continuously differentiable in } t\} .
$$

To establish our main theorem we need the following lemmas.

Lemma 1: [14] Let $\left(H_{1}\right)$ hold. Then,

(i) there exist constants $M \geq 1$ and $\omega \geq 0$ such that

$$
\|C(t)\| \leq N e^{\omega ! t \mid} \text { and }\left\|S(t)-S\left(t^{*}\right)\right\| \leq N\left|\int_{t}^{t^{*}} e^{\omega|s|} d s\right| \text { for } t, t^{*} \in \mathbb{R}
$$


(ii) $\quad S(t) X \subset E$ and $S(t) E \subset D(A)$ for $t \in \mathbb{R}$;

(iii) $\quad \frac{d}{d t} C(t) x=A S(t) x$ for $x \in E$ and $t \in \mathbb{R}$;

(iv) $\quad \frac{d^{2}}{d t^{2}} C(t) x=A C(t) x$ for $x \in D(A)$ and $t \in \mathbb{R}$.

Lemma 2: [14] Let $\left(H_{1}\right)$ hold, let $v: \mathbb{R} \rightarrow X$ be such that $v$ is continuously differentiable and let $q(t)=\int{ }_{0}^{t} S(t-s) v(s) d s$. Then,

$q$ is twice continuously differentiable and for $t \in \mathbb{R}, q(t) \in D(A)$,

$$
\begin{gathered}
q^{\prime}(t)=\int_{0}^{t} C(t-s) v(s) d s, \text { and } q^{\prime \prime}(t)=\int_{0}^{t} C(t-s) v^{\prime}(s) d s+C(t) v(0) \\
=A q(t)+v(t) .
\end{gathered}
$$

Lemma 3: (Schaefer Theorem [13]) Let $S$ be a convex subset of a normed linear space $Y$ and assume $0 \in S$. Let $F: S \rightarrow S$ be a completely continuous operator, and let

$$
\xi(F)=\{x \in S: x=\lambda F x \text { for some } 0<\lambda<1\} .
$$

Then either $\xi(F)$ is unbounded or $F$ has a fixed point.

We make the following assumptions:

$\left(H_{2}\right) \quad f(t, \cdot, \cdot): X \times X \rightarrow X$ is continuous for each $t \in J$ and the function $f(\cdot, x, y): J \rightarrow X$ is strongly measurable (see [12, p. 116]) for each $x, y \in X$.

$\left(H_{3}\right) \quad$ For every positive constant $k$ there exists $\alpha_{k} \in L^{1}(J)$ such that

$$
\sup _{\|x\|,\|y\| \leq k}\|f(t, x, y)\| \leq \alpha_{k}(t) \text { for almost all } t \in J
$$

$\left(H_{4}\right)$ There exists a continuous function $m: J \rightarrow[0, \infty)$ such that

$$
\|f(t, u, v)\| \leq m(t) \Omega(\|u\|+\|v\|), t \in J, u, v \in X
$$

where $\Omega:(0, \infty) \rightarrow(0, \infty)$ is a continuous nondecreasing function and

$$
M(T+1) \int_{0}^{T} m(s) d s<\int_{c}^{\infty} \frac{d s}{\Omega(s)}
$$

with

$$
\begin{aligned}
& c=\left(M+M^{*}\right)\left\|x_{0}\right\|+(1+T) M\left\|y_{0}\right\|+(1+T) M\|B\| T\left\|W^{-1}\right\| \\
& {\left[\left\|x_{1}\right\|+M\left\|x_{0}\right\|+M\left\|y_{0}\right\|+M T \int_{0}^{T} m(s) \Omega\left(\|x(s)\|+\left\|x^{\prime}(s)\right\|\right) d s\right],} \\
& M=\sup \{\|C(t)\|: t \in J\}, \text { and } M^{*}=\sup \{\|A S(t)\|: t \in J\} . \\
& \left(H_{5}\right) \quad B u(t) \text { is continuous. } \\
& \left(H_{6}\right) \text { The linear operator } W: L^{2}(J, U) \rightarrow X \text { defined by }
\end{aligned}
$$




$$
W u=\int_{0}^{T} S(T-s) B u(s) d s
$$

has a bounded invertible operator $W^{-1}: X \rightarrow L^{2}(J, U) / k e r W$.

$\left(H_{7}\right) \quad C(t), t>0$ is compact.

Then the system (1) has a mild solution of the form (see [9])

$$
\begin{aligned}
x(t)=C(t) x_{0}+ & S(t) y_{0}+\int_{0}^{t} S(t-s) f\left(s, x(s), x^{\prime}(s)\right) d s \\
& +\int_{0}^{t} S(t-s) B u(s) d s .
\end{aligned}
$$

Definition 2: The system (1) is said to be controllable on $J$ if for every $x_{0} \in D(A), y_{0} \in E$ and $x_{1} \in X$ there exists a control $u=L^{2}(J, U)$ such that the solution $x(\cdot)$ of $(1)$ satisfies $x(T)=x_{1}$.

\section{Main Result}

Theorem: Suppose $\left(H_{1}\right)-\left(H_{7}\right)$ hold. Then system $(1)$ is controllable on $J$.

Proof: Using $\left(H_{6}\right)$, for an arbitrary function $x(\cdot)$ we define the control

$$
u(t)=W^{-1}\left[x_{1}-C(T) x_{0}-S(T) y_{0}-\int_{0}^{T} S(T-s) f\left(s, x(s), x^{\prime}(s)\right) d s\right](t) .
$$

Using this control we will show that the operator defined by

$$
\begin{aligned}
(F x)(t) & =C(t) x_{0}+S(t) y_{0}+\int_{0}^{t} S(t-s) f\left(s, x(s), x^{\prime}(s)\right) d s \\
& +\int_{0}^{t} S(t-s) B W^{-1}\left[x_{1}-C(T) x_{0}-S(T) y_{0}\right. \\
& \left.-\int_{0}^{T} S(T-\theta) f\left(\theta, x(\theta), x^{\prime}(\theta)\right) d \theta\right](s) d s, \quad t \in J,
\end{aligned}
$$

has a fixed point. This fixed point is then a solution of Equation (2).

Clearly, $(F x)(T)=x_{1}$, which means that the control $u$ steers the system from the initial state $x_{0}$ to $x_{1}$ in time $T$, provided we obtain a fixed point of the nonlinear operator $F$. Consider the space $Z=C^{1}(J, X)$ with norm

$$
\|x\|^{*}=\max \left\{\|x\|,\left\|x^{\prime}\right\|\right\} \text {. }
$$

In order to study the controllability problem for the system (1) we apply Lemma 3 to 
the following system

$$
x^{\prime \prime}(t)=\lambda A x(t)+\lambda f\left(t, x(t), x^{\prime}(t)\right)+\lambda B u(t), t \in J, \lambda \in(0,1) .
$$

Let $x$ be a mild solution of system (3). Then from

$$
\begin{aligned}
x(t)= & \lambda\left(C(t) x_{0}+S(t) y_{0}\right)+\lambda \int_{0}^{t} S(t-s) f\left(s, x(s), x^{\prime}(s)\right) d s \\
& +\lambda \int_{0}^{t} S(t-s) B W^{-1}\left[x_{1}-C(T) x_{0}-S(T) y_{0}\right. \\
& \left.-\int_{0}^{T} S(T-\theta) f\left(\theta, x(\theta), x^{\prime}(\theta)\right) d \theta\right](s) d s
\end{aligned}
$$

we have

$$
\begin{aligned}
\|x(t)\| \leq & M\left\|x_{0}\right\|+M T\left\|y_{0}\right\|+M T \int_{0}^{t} m(s) \Omega\left(\|x(s)\|+\left\|x^{\prime}(s)\right\|\right) d s \\
& +M T^{2}\|B\| W^{-1} \|\left[\left\|x_{1}\right\|+M\left\|x_{0}\right\|+M T\left\|y_{0}\right\|\right. \\
& \left.+M T \int_{0}^{T} m(s) \Omega\left(\|x(s)\|+\left\|x^{\prime}(s)\right\|\right) d s\right] \\
\equiv & K_{1}+M T \int_{0}^{t} m(s) \Omega\left(\|x(s)\|+\left\|x^{\prime}(s)\right\|\right) d s .
\end{aligned}
$$

Denoting by $v(t)$ the right-hand side of the above inequality we have

$$
v(0)=K_{1}, \quad\|x(t)\| \leq v(t)
$$

and

$$
v^{\prime}(t)+\operatorname{MTm}(t) \Omega\left(\|x(t)\|+\left\|x^{\prime}(t)\right\|\right), t \in J
$$

On the other hand,

$$
\begin{aligned}
x^{\prime}(t)= & \lambda\left[A S(t) x_{0}+C(t) y_{0}\right]+\lambda \int_{0}^{t} C(t-s) f\left(s, x(s), x^{\prime}(s)\right) d s \\
& +\lambda \int_{0}^{t} C(t-s) B W^{-1}\left[x_{1}-C(T) x_{0}-S(T) y_{0}\right. \\
& \left.-\int_{0}^{T} S(T-\theta) f\left(\theta, x(\theta), x^{\prime}(\theta)\right) d \theta\right](s) d s .
\end{aligned}
$$


Thus we have

$$
\begin{gathered}
\left\|x^{\prime}(t)\right\| \leq M^{*}\left\|x_{0}\right\|+M\left\|y_{0}\right\|+M \int_{0}^{t} m(s) \Omega\left(\|x(s)\|+\left\|x^{\prime}(s)\right\|\right) d s \\
+M T\|B\|\left\|W^{-1}\right\|\left[\left\|x_{1}\right\|+M\left\|x_{0}\right\|+M T\left\|y_{0}\right\|\right. \\
\left.+M T \int_{0}^{T} m(s) \Omega\left(\|x(s)\|+\left\|x^{\prime}(s)\right\|\right) d s\right] \\
\equiv K_{2}+M \int_{0}^{t} m(s) \Omega\left(\|x(s)\|+\left\|x^{\prime}(s)\right\|\right) d s .
\end{gathered}
$$

Denoting by $r(t)$ the right-hand side of the above inequality we have

$$
r(0)=K_{2}, \quad\left\|x^{\prime}(t)\right\| \leq r(t)
$$

and

$$
r^{\prime}(t)=M m(t) \Omega\left(\|x(t)\|+\left\|x^{\prime}(t)\right\|\right), \quad t \in J
$$

Let

$$
w(t)=v(t)+r(t), \quad t \in J
$$

Then

$$
w(0)=v(0)+r(0)=c
$$

and

$$
\begin{gathered}
w^{\prime}(t)=v^{\prime}(t)+r^{\prime}(t) \\
\leq M T m(t) \Omega(w(t))+M m(t) \Omega(w(t)) \\
=M(T+1) m(t) \Omega(w(t)), \quad t \in J .
\end{gathered}
$$

This implies

$$
\int_{w(0)}^{w(t)} \frac{d s}{\Omega(s)} \leq M(T+1) \int_{0}^{T} m(s) d s<\int_{c}^{\infty} \frac{d s}{\Omega(s)}, \quad t \in J .
$$

The last inequality implies that there is a constant $K$ such that

$$
w(t)=v(t)+r(t) \leq K, \quad t \in J
$$

Then 


$$
\begin{gathered}
\|x(t)\| \leq v(t), \quad t \in J \\
\left\|x^{\prime}(t)\right\| \leq r(t), \quad t \in J
\end{gathered}
$$

and hence

$$
\|x\|^{*}=\max \left\{\|x\|,\left\|x^{\prime}\right\|\right\} \leq K
$$

where $K$ depends only on $T$ and on the functions $m$ and $\Omega$.

We shall now prove that the operator $F: Z \rightarrow Z$ defined by

$$
\begin{aligned}
(F x)(t) & =C(t) x_{0}+S(t) y_{0}+\int_{0}^{t} S(t-s) f\left(s, x(s), x^{\prime}(s)\right) d s \\
& +\int_{0}^{t} S(t-s) B W^{-1}\left[x_{1}-C(T) x_{0}-S(T) y_{0}\right. \\
& \left.-\int_{0}^{T} S(T-\theta) f\left(\theta, x(\theta), x^{\prime}(\theta)\right) d \theta\right](s) d s, \quad t \in J
\end{aligned}
$$

is a completely continuous operator.

Let $B_{k}=\left\{x \in Z,\|x\|^{*} \leq k\right\}$ for some $k \geq 1$. We first show that $F$ maps $B_{k}$ into an equicontinuous family. Let $x \in B_{k}$ and $t_{1}, t_{2} \in J$. Then if $0<t_{1}<t_{2} \leq T$,

$$
\begin{gathered}
\left\|(F x)\left(t_{1}\right)-(F x)\left(t_{2}\right)\right\| \leq\left\|C\left(t_{1}\right)-C\left(t_{2}\right)\right\|\left\|x_{0}\right\|+\left\|S\left(t_{1}\right)-S\left(t_{2}\right)\right\|\left\|y_{0}\right\| \\
+\left\|\int_{0}^{t_{1}}\left[S\left(t_{1}-s\right)-S\left(t_{2}-s\right)\right] f\left(s, x(s), x^{\prime}(s)\right) d s\right\| \\
\quad+\left\|\int_{t_{1}}^{t_{2}} S\left(t_{2}-s\right) f\left(s, x(s), x^{\prime}(s)\right) d s\right\| \\
+\| \int_{0}^{t_{1}}\left[S\left(t_{1}-s\right)-S\left(t_{2}-s\right)\right] B W^{-1}\left[x_{1}-C(T) x_{0}-S(T) y_{0}\right. \\
\left.\quad-\int_{0}^{T} S(T-\theta) f\left(\theta, x(\theta), x^{\prime}(\theta)\right) d \theta\right](s) d s \| \\
+\| \int_{t_{1}}^{t_{2}} S\left(t_{2}-s\right) B W-1\left[x_{1}-C(T) x_{0}-S(T) y_{0}\right. \\
\left.\quad-\int_{0}^{T} S(T-\theta) f\left(\theta, x(\theta), x^{\prime}(\theta)\right) d \theta\right](s) d s \| \\
\leq C\left(t_{1}\right)^{-} C\left(t_{2}\right)\|\| x_{0}\|+\| S\left(t_{1}\right)-S\left(t_{2}\right)\|\| y_{0} \|
\end{gathered}
$$




$$
\begin{gathered}
+\int_{0}^{t_{1}}\left\|S\left(t_{1}-s\right)-S\left(t_{2}-s\right)\right\| \alpha_{k}(s) d s \\
+\int_{t_{1}}^{t_{2}}\left\|S\left(t_{2}-s\right)\right\| \alpha_{k}(s) d s \\
+\int_{0}^{t_{1}}\left\|S\left(t_{1}-s\right)-S\left(t_{2}-s\right)\right\|\|B\|\left\|W^{-1}\right\|\left[\left\|x_{1}\right\|+M\left\|x_{0}\right\|+M T\left\|y_{0}\right\|\right. \\
\left.+M T \int_{0}^{T} \alpha_{k}(\theta) d \theta\right] d s \\
+\int_{t_{1}}^{t_{2}}\left\|S\left(t_{2}-s\right)\right\|\|B\| W-1 \|\left[\left\|x_{1}\right\|+M\left\|x_{0}\right\|+M T\left\|y_{0}\right\|\right. \\
\left.+M T \int_{0}^{T} \alpha_{k}(\theta) d \theta\right] d s,
\end{gathered}
$$

and similarly,

$$
\begin{aligned}
& \left\|(F x)^{\prime}\left(t_{1}\right)-(F x)^{\prime}\left(t_{2}\right)\right\| \leq\left\|C^{\prime}\left(t_{1}\right)-C^{\prime}\left(t_{2}\right)\right\|\left\|x_{0}\right\|+\left\|S^{\prime}\left(t_{1}\right)-S^{\prime}\left(t_{2}\right)\right\|\left\|y_{0}\right\| \\
& +\left\|\int_{0}^{t_{1}}\left[C\left(t_{1}-s\right)-C\left(t_{2}-s\right)\right] f\left(s, x(s), x^{\prime}(s)\right) d s\right\| \\
& +\left\|\int_{t_{1}}^{t_{2}} C\left(t_{2}-s\right) f\left(s, x(s), x^{\prime}(s)\right) d s\right\| \\
& +\| \int_{0}^{t_{1}}\left[C\left(t_{1}-s\right)-C\left(t_{2}-s\right)\right] B W^{-1}\left[x_{1}-C(T) x_{0}-S(T) y_{0}\right. \\
& \left.-\int_{0}^{T} S(T-\theta) f\left(\theta, x(\theta), x^{\prime}(\theta)\right) d \theta\right](s) d s \| \\
& +\| \int_{t_{1}}^{t_{2}} C\left(t_{2}-s\right) B W^{-1}\left[x_{1}-C(T) x_{0}-S(T) y_{0}\right. \\
& \left.-\int_{0}^{T} S(T-\theta) f\left(\theta, x(\theta), x^{\prime}(\theta)\right) d \theta\right](s) d s \| \\
& \leq\left\|A\left(S\left(t_{1}\right)-S\left(t_{2}\right)\right)\right\|\left\|x_{0}\right\|+\left\|C\left(t_{1}\right)-C\left(t_{2}\right)\right\|\left\|y_{0}\right\| \\
& +\int_{0}^{t_{1}}\left\|C\left(t_{1}-s\right)-C\left(t_{2}-s\right)\right\| \alpha_{k}(s) d s
\end{aligned}
$$




$$
\begin{gathered}
\quad+\int_{t_{1}}^{t_{2}}\left\|C\left(t_{2}-s\right)\right\| \alpha_{k}(s) d s \\
+\int_{0}^{t_{1}}\left\|C\left(t_{1}-s\right)-C\left(t_{2}-s\right)\right\|\|B\|\left\|W^{-1}\right\|\left[\left\|x_{1}\right\|+M\left\|x_{0}\right\|+M T\left\|y_{0}\right\|\right. \\
\left.+M T \int_{0}^{T} \alpha_{k}(\theta) d \theta\right] d s \\
+\int_{t_{1}}^{t_{2}}\left\|C\left(t_{2}-s\right)\right\|\|B\| W-1 \|\left[\left\|x_{1}\right\|+M\left\|x_{0}\right\|+M T\left\|y_{0}\right\|\right. \\
\left.+M T \int_{0}^{T} \alpha_{k}(\theta) d \theta\right] d s .
\end{gathered}
$$

The right-hand side of (4) and (5) are independent of $y \in B_{k}$ and tends to zero as $t_{2}-t_{1} \rightarrow 0$, since $C(t)$ and $S(t)$ are uniformly continuous for $t \in J$. The compactness of $C(t)$ and $S(t)$ for $t>0$ imply the continuity in the uniform operator topology (or norm topology, see [12, p. 182]). Furthermore, the compactness of $S(t)$ follows from that of $C(t)$ (see [15]).

Thus $F$ maps $B_{k}$ into an equicontinuous family of functions. It is easy to see that the family $F B_{k}$ is uniformly bounded.

Next we show that $\overline{F B_{k}}$ is compact. Since we have shown that $F B_{k}$ is an equicontinuous collection, it suffices by the Arzela-Ascoli theorem to show that $F$ maps $B_{k}$ into a precompact set in $X$.

Let $0<t \leq T$ be fixed and let $\epsilon$ be a real number satisfying $0<\epsilon<t$. For $x \in B_{k}$, we define

$$
\begin{aligned}
\left(F_{\epsilon} x\right)(t) & =C(t) x_{0}+S(t) y_{0}+\int_{0}^{t-\epsilon} S(t-s) f\left(s, x(s), x^{\prime}(s)\right) d s \\
& +\int_{0}^{t-\epsilon} S(t-s) B W^{-1}\left[x_{1}-C(T) x_{0}-S(T) y_{0}\right. \\
& \left.-\int_{0}^{T} S(T-\theta) f\left(\theta, x(\theta), x^{\prime}(\theta)\right) d \theta\right](s) d s .
\end{aligned}
$$

Since $C(t)$ and $S(t)$ are compact operators, the set $Y_{\epsilon}(t)=\left\{\left(F_{\epsilon} x\right)(t): x \in B_{k}\right\}$ is precompact in $X$ for every $\epsilon, 0<\epsilon<t$. Moreover, for every $x \in B_{k}$, we have that

$$
\begin{gathered}
\left\|(F x)(t)-\left(F_{\epsilon} x\right)(t)\right\| \leq \int_{t-\epsilon}^{t}\left\|S(t-s) f\left(s, x(s), x^{\prime}(s)\right)\right\| d s \\
+\int_{t-\epsilon}^{t} \| S(t-s) B W^{-1}\left[x_{1}-C(T) x_{0}-S(T) y_{0}\right.
\end{gathered}
$$




$$
\begin{gathered}
\left.-\int_{0}^{T} S(T-\theta) f\left(\theta, x(\theta), x^{\prime}(\theta)\right) d \theta\right](s) \| d s \\
\leq \int_{t-\epsilon}^{t}\|S(t-s)\| \alpha_{k}(s) d s \\
+\int_{t-\epsilon}^{t}\|S(t-s)\|\|B\|\left\|W^{-1}\right\|\left[\left\|x_{1}\right\|+M\left\|x_{0}\right\|+M T\left\|y_{0}\right\|\right. \\
\left.\left.+M T \int_{0}^{T} \alpha_{k}(\theta)\right) d \theta\right] d s
\end{gathered}
$$

and

$$
\begin{gathered}
\left\|(F x)^{\prime}(t)-\left(F_{\epsilon} x\right)^{\prime}(t)\right\| \leq \int_{t-\epsilon}^{t}\left\|C(t-s) f\left(s, x(s), x^{\prime}(s)\right)\right\| d s \\
+\int_{t-\epsilon}^{t} \| C(t-s) B W^{-1}\left[x_{1}-C(T) x_{0}-S(T) y_{0}\right. \\
\left.-\int_{0}^{T} S(T-\theta) f\left(\theta, x(\theta), x^{\prime}(\theta)\right) d \theta\right](s) \| d s . \\
\leq \int_{t-\epsilon}^{t}\|C(t-s)\| \alpha_{k}(s) d s \\
+\int_{t-\epsilon}^{t}\|C(t-s)\|\|B\|\|W-1\|\left[\left\|x_{1}\right\|+M\left\|x_{0}\right\|+M T\left\|y_{0}\right\|\right. \\
\left.+M T \int_{0}^{T} \alpha_{k}(\theta) d \theta\right] d s .
\end{gathered}
$$

Therefore, there are precompact sets arbitrarily close to the set $\left\{(F x)(t): x \in B_{k}\right\}$. Hence the set $\left\{(F x)(t): x \in B_{k}\right\}$ is precompact in $X$.

It remains to show that $F: Z \rightarrow Z$ is continuous. Let $\left\{x_{n}\right\}_{0}^{\infty} \subseteq Z$ with $x_{n} \rightarrow x$ in $Z$. Then there is an integer $q$ such that $\left\|x_{n}(t)\right\| \leq q,\left\|x_{n}^{\prime}(t)\right\| \leq q$ for all $n$ and $t \in J$, so $\|x(t)\| \leq q,\left\|x^{\prime}(t)\right\| \leq q$ and $x, x^{\prime} \in Z$. By $\left(H_{2}\right)$

$$
f\left(t, x_{n}(t), x_{n}^{\prime}(t)\right) \rightarrow f\left(t, x(t), x^{\prime}(t)\right)
$$

for each $t \in J$ and since

$$
\left\|f\left(t, x_{n}(t), x_{n}^{\prime}(t)\right)-f\left(t, x(t), x^{\prime}(t)\right)\right\| \leq 2 \alpha_{q}(t),
$$

we have by the dominated convergence theorem that

$$
\left\|F x_{n}-F x\right\|=\sup _{t \in J} \| \int_{0}^{t} S(t-s)\left[f\left(s, x_{n}(s), x_{n}^{\prime}(s)\right)-f\left(s, x(s), x^{\prime}(s)\right)\right] d s
$$




$$
\begin{gathered}
-\int_{0}^{t} S(t-s) B W^{-1} \\
\times \int_{0}^{T} S(T-\theta)\left[f\left(\theta, x_{n}(\theta), x_{n}^{\prime}(\theta)\right)-f\left(\theta, x(\theta), x^{\prime}(\theta)\right)\right] d \theta d s \| \\
\leq \int_{0}^{T}\left\|S(t-s)\left[f\left(s, x_{n}(s), x_{n}^{\prime}(s)\right)-f\left(s, x(s), x^{\prime}(s)\right)\right]\right\| d s \\
+\int_{0}^{T} \| S(t-s) B W^{-1} \\
\times \int_{0}^{T} S(T-\theta)\left[f\left(\theta, x_{n}(\theta), x_{n}^{\prime}(\theta)\right)-f\left(\theta, x(\theta), x^{\prime}(\theta)\right)\right] d \theta \| d s \rightarrow 0
\end{gathered}
$$

and

$$
\begin{aligned}
\|\left(F x_{n}\right)^{\prime}- & (F x)^{\prime}\left\|=\sup _{t \in J}\right\| \int_{0}^{t} C(t-s)\left[f\left(s, x_{n}(s), x_{n}^{\prime}(s)\right)-f\left(s, x(s), x^{\prime}(s)\right)\right] d s \\
& -\int_{0}^{t} C(t-s) B W^{-1} \\
& \times \int_{0}^{T} S(T-\theta)\left[f\left(\theta, x_{n}(\theta), x_{n}^{\prime}(\theta)\right)-f\left(\theta, x(\theta), x^{\prime}(\theta)\right)\right] d \theta d s \| \\
\leq & \quad+\int_{0}^{T}\left\|C(t-s)\left[f\left(s, x_{n}(s), x_{n}^{\prime}(s)\right)-f\left(s, x(s), x^{\prime}(s)\right)\right]\right\| d s \\
& \times \int_{0}^{T} S(T-\theta)\left[f\left(\theta, x_{n}(\theta), x_{n}^{\prime}(\theta)\right)-f\left(\theta, x(\theta), x^{\prime}(\theta)\right)\right] d \theta \| d s \rightarrow 0 .
\end{aligned}
$$

Thus $F$ is continuous. This completes the proof that $F$ is completely continuous.

Finally, the set $\zeta(F)=\{x \in Z: x=\lambda F x, \lambda \in(0,1)\}$ is bounded as we proved it in the first step. Consequently, by Schaefer's theorem, the operator $F$ has a fixed point in $Z$. This means that any fixed point of $F$ is a mild solution of (1) on $J$ satisfying $(F x)(t)=x(t)$. Thus the system (1) is controllable on $J$.

\section{Example}

Consider the partial differential equation

$$
\begin{gathered}
z_{t t}(y, t)=z_{y y}(y, t)+\sigma\left(t, z(y, t), z_{t}(y, t)\right)+\mu(y, t) \\
z(0, t)=z(\pi, t)=0
\end{gathered}
$$




$$
z(y, 0)=z_{0}(y), \quad z_{t}(y, 0)=z_{1}(y), \quad 0<y<\pi, t \in J=[0, T]
$$

Now we have to show that there exists a control $\mu$ which steers (6) from any specified initial state to the final state in a Banach space $X$.

Let $X=L^{2}[0, \pi]$ and let $A: X \rightarrow X$ be defined by

$$
A w=w^{\prime \prime}, \quad w \in D(A)
$$

where $D(A)=\left\{w \in X: w, w^{\prime}\right.$ are absolutely continuous, $\left.w^{\prime \prime} \in X, w(0)=w(\pi)=0\right\}$.

Then,

$$
A w=\sum_{n=1}^{\infty}-n^{2}\left(w, w_{n}\right) w_{n}, w \in D(A)
$$

where $w_{n}(s)=\sqrt{2 / \pi} \sin n s, n=1,2,3, \ldots$ is the orthogonal set of eigenvalues of $A$.

It can be easily shown that $A$ is the infinitesimal generator of a strongly continuous cosine family $C(t), t \in \mathbb{R}$, in $X$ given by

$$
C(t) w=\sum_{n=1}^{\infty} \cos n t\left(w, w_{n}\right) w_{n}, w \in X,
$$

and that the associated sine family is given by

$$
S(t) w=\sum_{n=1}^{\infty} \frac{1}{n} \sin n t\left(w, w_{n}\right) w_{n}, \quad w \in X .
$$

Let $f: J \times X \times X \rightarrow X$ be define by

$$
f(t, v, w)(y)=\sigma(t, v(y), w(y)), v, w \in X, y \in[0, \pi]
$$

where $\sigma: J \times[0, \pi] \times[0, \pi] \rightarrow[0, \pi]$ is continuous and strongly measurable.

Let $u: J \rightarrow U \subset X$ be defined by

$$
(u(t))(y)=\mu(y, t), \quad y \in[0, \pi],
$$

where $\mu:[0, \pi] \times J \rightarrow[0, \pi]$ is continuous.

Assume that there exists a bounded invertible operator $W^{-1}$ (with range $\left.L^{2}(J, U) / k e r W\right)$ such that

$$
W u=\int_{0}^{T} S(T-s) u(s) d s .
$$

Further, the function $\sigma$ satisfies the following condition:

(H) There exists a continuous function $p: J \rightarrow[0, \infty)$ such that

$$
\|\sigma(t, u, v)\| \leq p(t) \Omega(\|u\|+\|v\|), \quad t \in J, u, v \in X
$$

where $\Omega:(0, \infty) \rightarrow(0, \infty)$ is a continuous nondecreasing function and

where $c$ is a known constant.

$$
M(T+1) \int_{0}^{T} p(s) d s<\int_{c}^{\infty} \frac{d s}{\Omega(s)}
$$


With this choice of $A, f$ and $B=I,(1)$ is an abstract formulation of (6). Furthermore, all the conditions stated in the above theorem are satisfied. Hence, system (6) is controllable on $J$.

\section{Acknowledgement}

The work of the first author is supported by CSIR, New Delhi, India (Grant No: 25(89)97-EMR-II).

\section{References}

[1] Balachandran, K., Dauer, J.P. and Balasubramaniam, P., Controllability of nonlinear integrodifferential systems in Banach spaces, J. of Optim. Theory and Appl. 84 (1995), 83-91.

[2] Bochenek, J., An abstract nonlinear second order differential equation, Annales Polonici Math. Lv. 54 (1991), 155-166.

[3] Chukwu, E.N. and Lenhart, S.M., Controllability questions for nonlinear systems in abstract spaces, J. of Optim. Theory and Appl. 68 (1991), 437-462.

[4] Fitzgibbon, W.E., Global existence and boundedness of solutions to the extensible beam equation, SIAM J. of Math. Anal. 13 (1982), 739-745.

[5] MacCamy, R.C., A model for one-dimensional nonlinear viscoelasticity, Quart. of Appl. Math 35 (1977), 21-33.

[6] MacCamy, R.C., An integrodifferential equation with applications in heat flow, Quart. of Appl. Math 35 (1977), 1-19.

[7] Naito, K., Controllability of semilinear control systems dominated by the linear part, SIAM J. on Contr. and Optim. 25 (1987), 715-722.

[8] Naito, K., On controllability for a nonlinear Volterra equation, Nonl. Anal.: Theory, Methods and Appl. 18 (1992), 99-108.

[9] Ntouyas, S.K. and Tsamatos, P.Ch., Global existence for second order semilinear ordinary and delay integrodifferential equations with nonlocal conditions, Appl. Anal. 67 (1997), 245-257.

[10] Park, J.Y. and Han, H.K., Controllability for some second order differential equations, Bull. of the Korean Math. Soc. 34 (1997), 411-419.

[11] Quinn, M.D. and Carmichael, N., An approach to nonlinear control problems using fixed point methods, degree theory, and pseudo-inverses, Num. Funct. Anal. and Optim. 7 (1984-1985), 197-219.

[12] Reed, M. and Simon, B., Functional Analysis, Academic Press, New York 1973.

[13] Schaefer, H., Uber die methode der a priori schranken, Math. Annalem 129 (1955), 415-416.

[14] Travis, C.C. and Webb, G.F., Cosine families and abstract nonlinear second order differential equations, Acta Math. Acad. Scient. Hung. 32 (1978), 75-96.

[15] Travis, C.C. and Webb, G.F., Compactness, regularity and uniform continuity properties of strongly continuous cosine families, Houston J. of Math. 3 (1977), 555-567. 


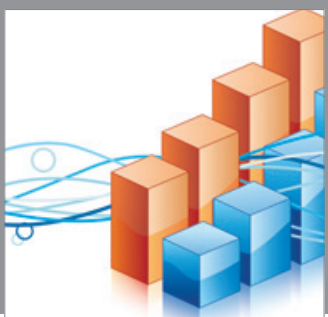

Advances in

Operations Research

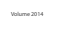

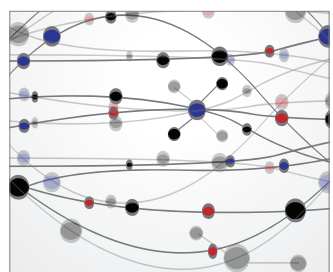

\section{The Scientific} World Journal
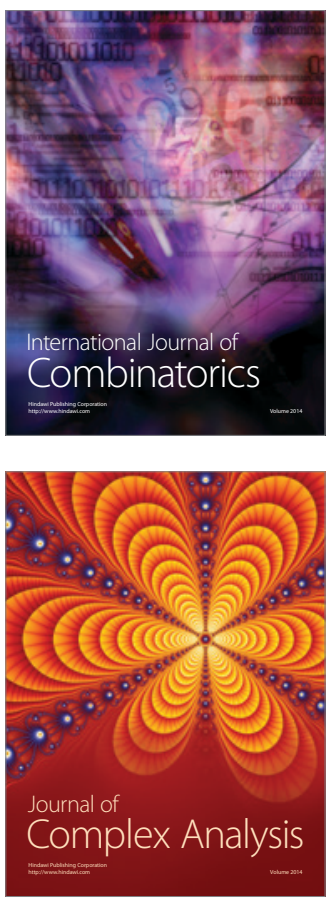

International Journal of

Mathematics and

Mathematical

Sciences
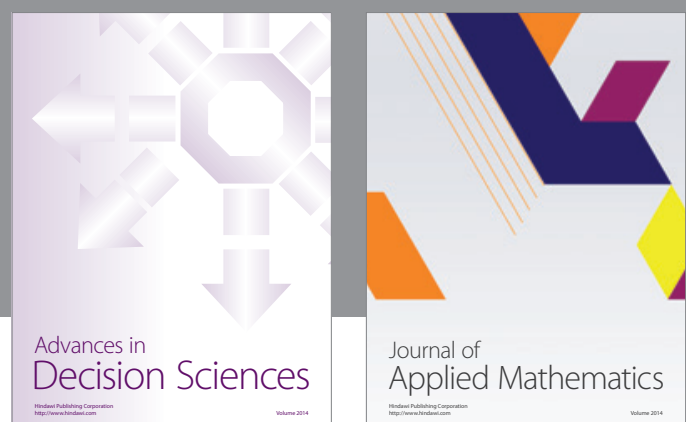

Journal of

Applied Mathematics
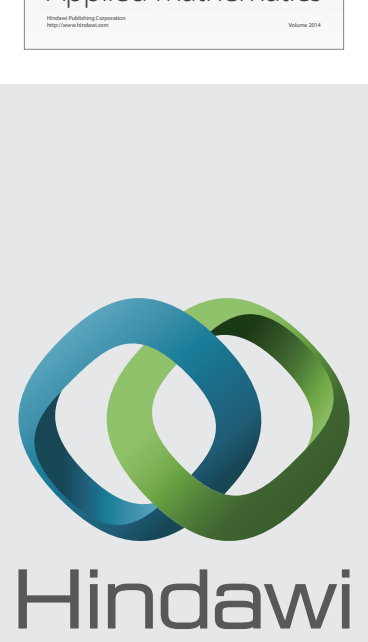

Submit your manuscripts at http://www.hindawi.com
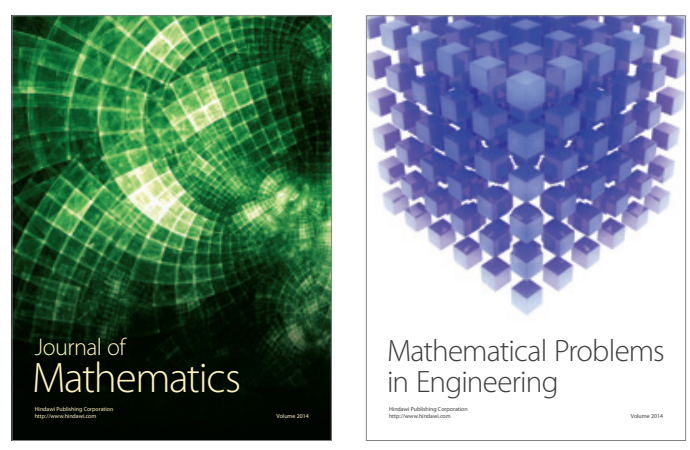

Mathematical Problems in Engineering
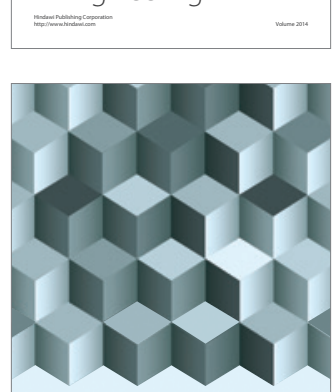

Journal of

Function Spaces
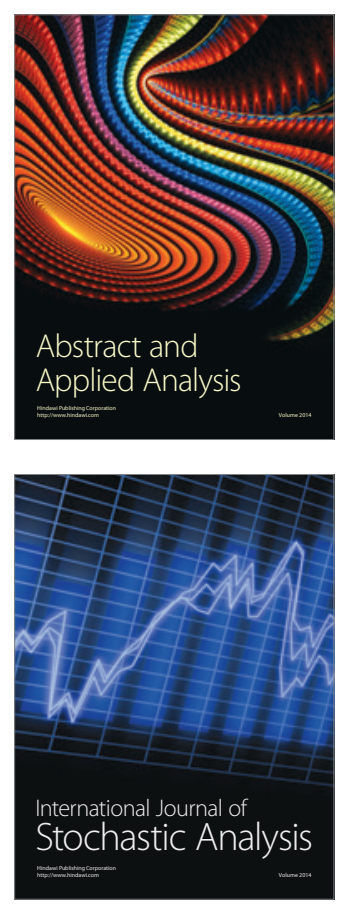

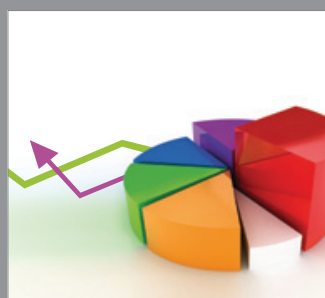

ournal of

Probability and Statistics

Promensencen
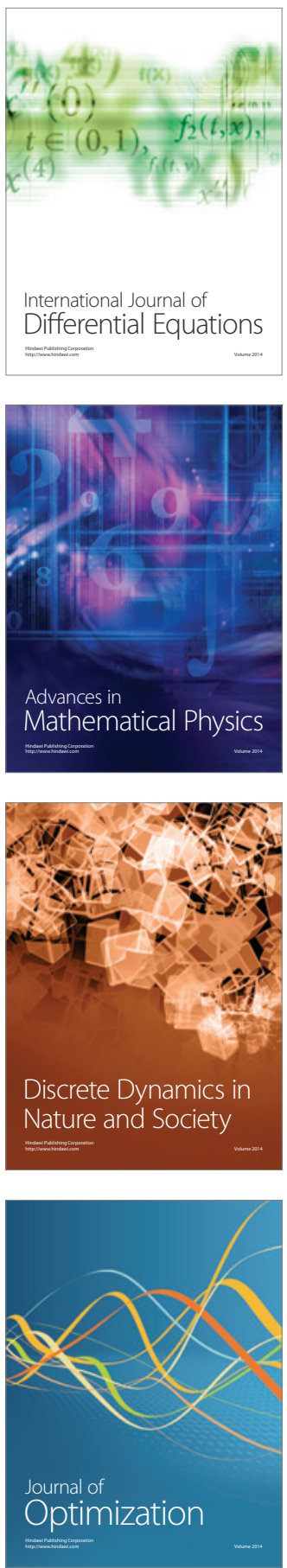